\title{
Correction to: A DELPHI consensus statement on antiplatelet management for intracranial stenting due to underlying atherosclerosis in the setting of mechanical thrombectomy
}

\author{
Mayank Goyal ${ }^{1}$. $\cdot$ Kirill Orlov ${ }^{2}$ Mary E. Jensen ${ }^{3}$. Allan Taylor ${ }^{4}$. Charles Majoie ${ }^{5} \cdot$ Mahesh Jayaraman $^{6}$. \\ Jianmin Liư ${ }^{7}$ Geneviève Milot $^{8}$ - Patrick Brouwer ${ }^{9,10}$. Shinichi Yoshimura ${ }^{11}$. Felipe Albuquerque ${ }^{12} \cdot$ Adam Arthur $^{13}$. \\ David Kallmes $^{14}$. Nobuyuki Sakai ${ }^{15}$. Justin F. Fraser ${ }^{16}$. Raul Nogueira ${ }^{17}$. Pengfei Yang ${ }^{7}$. Franziska Dorn ${ }^{18}$. \\ Lucie Thibault $^{19} \cdot$ Jens Fiehler $^{20} \cdot$ René Chapot $^{21} \cdot$ Johanna Maria Ospel $^{22,23}$
}

Published online: 14 June 2021

(c) Springer-Verlag GmbH Germany, part of Springer Nature 2021

Correction to: Neuroradiology (2021) 63:627-632 https://doi.org/10.1007/s00234-020-02556-z

The publisher would like to correct an error in Supplementary table I: Consensus recommendations for the dosage of GPIIb/IIIa receptor inhibitors*

Please note the Eptifibatide dosing is incorrect. The correct dose is $2 \mathrm{mcg} / \mathrm{kg} / \mathrm{min}$.

Publisher's note Springer Nature remains neutral with regard to jurisdictional claims in published maps and institutional affiliations.

The original article can be found online at https://doi.org/10.1007/ s00234-020-02556-z.

Mayank Goyal

mgoyal@ucalgary.ca

1 Departments of Radiology and Clinical Neurosciences, Foothills Medical Centre, University of Calgary, 1403 29th St. NW, Calgary, AB T2N2T9, Canada

2 Meshalkin National Medical Research Center, Novosibirsk, Russian Federation

3 Departments of Neurological Surgery, Radiology and Medical Imaging, UVA Health, Charlottesville, VA, USA

4 Groote Schuur Hospital, University of Cape Town, Cape Town, South Africa

5 Department of Radiology and Nuclear Medicine, Amsterdam University Medical Centers, location AMC, Amsterdam, The Netherlands

6 Departments of Diagnostic Imaging, Neurology and Neurosurgery, Warren Alpert School of Medicine at Brown University, Providence, RI, USA

7 Department of Neurosurgery, Changhai Hospital Naval Medical University, Shanghai, China

8 Department of Neurosurgery, CHU de Québec, Quebec City, Canada

9 Department of Interventional Neuroradiology, Karolinksa Hospital, Stockholm, Sweden

10 University NeuroVascular Center 'UNVC', Leiden University Medical Center, Haaglanden Medical Center, Leiden, Netherlands

11 Department of Neurosurgery, Hyogo College of Medicine, 1-1 Mukogawa, Nishinomiya, Hyogo, Japan 
12 Department of Neurosurgery, Barrow Neurological Institute, Phoenix, USA

13 Department of Neurosurgery, Semmes-Murphey Clinic/ University of TN, Memphis, TN, USA

14 Department of Radiology, Mayo Clinic, Rochester, USA

15 Department of Neurosurgery, Kobe City Medical Center General Hospital, Kobe, Japan

16 Departments of Neurosurgery, Neurology, Radiology, and Neuroscience, University of Kentucky, Lexington, KY, USA

17 Marcus Stroke \& Neuroscience Center, Grady Memorial Hospital and Department of Neurology, Emory University School of Medicine, Atlanta, GA, USA
18 Department of Neuroradiology, University Hospital Bonn, Bonn, Germany

19 WFITN, Paris, France

20 Department of Diagnostic and Interventional Neuroradiology, University Medical Center Hamburg-Eppendorf, Hamburg, Germany

21 Department of Neuroradiology, Alfred Krupp Krankenhaus Essen, Essen, Germany

22 Department of Clinical Neurosciences, University of Calgary, Calgary, Canada

23 Department of Neuroradiology, University Hospital of Basel, Basel, Switzerland 